\title{
Desemprego, autoestima e satisfação com a vida: estudo exploratório realizado em Portugal com beneficiários do Rendimento Social de Inserção
} Unemployment, Self-esteem, and Life Satisfaction: an exploratory study in Portugal with beneficiaries of the Social Inclusion Income. El desempleo, la autoestima y la satisfacción con la vida: un estudio exploratorio en Portugal con los beneficiarios del Rendimiento Social de Inserción.

\author{
» Rita Manuela BARROS ${ }^{1}$ (Instituto Piaget) \\ » Ana Sofia MOREIRA (Instituto Piaget)
}

Esta investigação pretende relacionar dois conceitos de natureza psicológica integrados no sistema do self a autoestima e a satisfação com a vida de adultos portugueses em situação de desemprego. Face à atual conjuntura socioeconômica, as taxas de desemprego têm disparado para valores históricos, havendo necessidade de conhecer de que forma esse fenômeno se relaciona com a percepção dos sujeitos na avaliação de si próprios e de satisfação com suas vidas. O estudo de natureza correlacional é constituído por 80 sujeitos em situação de desemprego, inscritos em um centro de emprego e que recebem proteção social por meio da atribuição do Rendimento Social de Inserção (RSI). Os resultados apontam para correlações positivas significativas entre a autoestima e a satisfação com a vida, a idade e a duração da situação de desemprego, a idade e a duração do período de atribuição do RSI e, ainda, esse período e a duração da situação de desemprego. Foi registrada uma correlação negativa e significativa entre a autoestima e a duração da situação de desemprego. Conclui-se globalmente que a duração da situação de desemprego do sujeito afeta sua autoestima e que a proteção social, como garantia de subsistência primária, não se relaciona com as variáveis do sistema do self em estudo, mais sofisticadas e com outras necessidades implícitas. $\mathrm{O}$ estudo aponta para a atenção que deverá ser dirigida aos desempregados de longa duração, mais velhos e, portanto, que recebem proteção social há mais tempo.

Palavras-chave:

Desemprego; inserção social; autoestima; satisfação com a vida.

Abstract

This research aims to relate two psychological concepts integrated in the self system, namely, selfesteem and life satisfaction of unemployed Portuguese adults. Given the current socio-economic situation, unemployment rates have been increasing to historical levels, raising the need to know how this phenomenon relates to the perception of self-evaluation and of the evaluation of life satisfaction. This is a correlational study based on a sample of 80 unemployed subjects registered in a job center and who receive social protection through the Social Inclusion Income (SII). The results show significant positive correlations between self-esteem and life satisfaction, between the subjects' age and the duration of the unemployment situation, between the subjects' age and the duration of the period during which they receive the SII, and even between this period and the duration of unemployment. There was only one significant negative correlation between self-esteem and the duration of unemployment. We conclude that the overall duration of the unemployment situation affects the self-esteem and that social protection, as a primary means of subsistence, is not related to the variables of the self system under study, which are more sophisticated and with other implied needs. The study suggests that attention should be directed to the long-term unemployed subjects, who are older and therefore have been receiving social protection for a longer period of time.

Keywords:

Unemployment; social inclusion; self-esteem; life satisfaction.

\footnotetext{
1 Doutora em Ciências da Educação. Research in Education and Community Intervention (RECI). Instituto Piaget. Endereço para contato - Escola Superior de Saúde Jean Piaget I Vila Nova de Gaia

Alameda Jean Piaget- 4405-678 Gulpilhares, Vila Nova de Gaia - Portugal.

E-mail: rita.barros@gaia.ipiaget.pt
} 
Esta investigación pretende comparar dos conceptos de naturaleza psicológica, integrados en el sistema del self, a saber, la autoestima y la satisfacción con la vida de adultos portugueses que se encuentran desempleados. Dado el actual contexto socio-económico, las tasas de desempleo se han disparado a niveles históricos, haciendo necesario conocer cómo este fenómeno se relaciona con la percepción de los sujetos en la evaluación de sí mismos y en la evaluación que hacen de la satisfacción con su vida. El estudio, de naturaleza correlacional, comprendió 80 sujetos que estaban desempleados, registrados en un centro de empleo y que recibían protección social por medio de la concesión del Rendimiento Social de Inserción (RSI). Los resultados muestran correlaciones significativas positivas entre la autoestima y la satisfacción con la vida, entre la edad y la duración del desempleo, entre la edad y la duración del período de recibimiento del RSI y entre este período y la duración de la situación de desempleo. Se registró una correlación negativa y significativa entre la autoestima y la duración de la situación de desempleo. Se concluyó que, globalmente, la duración de la situación de desempleo del sujeto afecta su autoestima y que la protección social, como garantía de subsistencia primaria no se relaciona con las variables del sistema del self en este estudio, más sofisticadas y otras necesidades implícitas. El estudio señala que se deberá prestar atención a las personas que están desempleadas por largos periodos, que son mayores y que, por lo tanto, reciben la protección social por más tiempo.

Palabras-clave:

Desempleo; inclusión social; autoestima; satisfacción con la vida.

A

atual crise econômica e social que assola a Europa desencadeou novas formas de inadaptação e de exclusão social, cujas consequências psicológicas não podem nem devem ser subestimadas (Luque, 1992; Sennett, 2000). Uma das formas de exclusão social mais relevantes nesse contexto é o desemprego (Costa, 1998; Costa, Baptista, Perista, \& Carrilho, 2008), efeito colateral decepcionante da expansão da globalização (Stiglitz, 2004) que se traduz na medida da força de trabalho disponível que se encontra sem emprego (Carvalho, 2008).

A centralidade do emprego e o entendimento do trabalho como eixo das relações sociais sempre foram questões de importância indiscutível em relação ao cumprimento das funções psicossociais de bem-estar (Álvaro, 1992; Álvaro, Torregrosa \& Luque, 1992; Blanch, 1990). Segundo Argolo e Araújo (2004), "quanto mais prolongado for o período de desemprego, maior será a deterioração do bem-estar psicológico dos trabalhadores, respeitado um ponto de saturação dos efeitos do desemprego, onde decrescerá o nível de deterioração do bem-estar psicológico" (p.171). Esta é, igualmente, a conclusão de outros estudos, com particular enfoque no contexto português. Caleiro (2012) sublinha não apenas a correlação negativa entre a dimensão subjetiva da felicidade e a situação de desemprego em Portugal, tomando como referência o período entre 1985 e 2010, mas a relação causal entre as variáveis, assinalando ainda o impacto da trajetória do desemprego, ou seja, da continuidade temporal da situação de desemprego, na dimensão subjetiva da experiência de felicidade.

A situação de desemprego não se circunscreve à privação da fonte de rendimento e, por consequência, à questão da subsistência. Estar desempregado compromete os vínculos sociais, as redes de relações interpessoais que o emprego proporciona, o sentimento de participação na vida econômica, as rotinas e a estruturação do tempo, os objetivos pessoais, o estatuto, o reconhecimento e a identidade social. Ao mesmo tempo, promove a insegurança, o sentimento de inutilidade, a vergonha social, o isolamento e a alteração da dinâmica familiar em função da posição que o indivíduo desempregado ocupa na família (Pereira \& Brito, 2006). "A posição de desempregado é apresentada como uma posição de menor prestígio, de segunda categoria, não providenciando uma verdadeira pertença ao social" (Caldas, 2000, como citado em Machado, 2003, p. 53). Acresce ainda o impacto do desemprego no bem-estar psicológico, no que diz respeito à imergência de patologia depressiva (Vieira, 2014).

Em Portugal, o Código de Trabalho em vigor apresenta as novas modalidades de despedimento contempladas na revisão de 2009: "despedimento por inadaptação" e "despedimento por extinção do posto de trabalho” (Lei n. ${ }^{\circ}$ 7/2009). Alterações posteriores incluídas nesse código, ao mesmo tempo em que ampliaram a liberdade das empresas para despedir, reduziram o montante das compensações pela cessação do contrato de trabalho (Lei no 53/2011; Lei no 23/2012). A proteção social aos desempregados é desencadeada pela atribuição do RSI, que se trata de um mecanismo de combate à pobreza que garante aos cidadãos e às famílias recursos para suprir suas necessidades mínimas por meio de prestação monetária, favorecendo a progressiva inserção social e profissional (Lei no 13/2003, de 21 de Maio, retificada pela Declaração retificativa nº7/2003, de 29 de Maio). Segundo o Instituto de Segurança Social, que regula a acessibilidade ao RSI, em maio de 2013

Rev. Psicol., Organ. Trab., abr-jun 2015, vol. 15 num. 2 
existiam, em Portugal, 272.062 beneficiários, em face de um total de 6.870 .043 portugueses em idade ativa, entre 15 e 65 anos (Pordata, n.d.a).

Essa conjuntura urge minimizar os efeitos nefastos do desemprego, pois são conhecidas as suas relações com a perda de autoestima, a deterioração psicológica geral e outras manifestações psicopatológicas (Álvaro, 1992; Álvaro \& Garrido, 2002; Banks, 1992; Buendía, 2001; Ripoll, Rodriguez, \& Peiró, 1996). Assim, as iniciativas de reintegração dos desempregados no mercado de trabalho são desejáveis não apenas por razões de ordem econômica, mas como estratégia de promoção da coesão social, da saúde e do bem-estar dos indivíduos.

Com esse propósito, a investigação no âmbito da psicologia poderá contribuir para a compreensão das implicações da situação de desemprego na vivência interna do sujeito. A autoestima e a satisfação com a vida resultam de processos avaliativos dirigidos a si mesmo, e sua relevância prende-se com o impacto que têm na forma como o sujeito lida com as circunstâncias de vida, especialmente em momentos de crise.

A autoestima se refere à avaliação, positiva ou negativa, que o indivíduo constrói sobre si mesmo (Alcántara, 1997), a qual condiciona sua forma de pensar, agir e sentir. Construída ao longo da história de vida e a partir das relações que se estabelecem com outros significativos (Cavalcanti, 2007), a autoestima integra o sistema do self (Janeiro \& Marques, 1999), definindo-se como o sentimento do indivíduo de se considerar globalmente uma pessoa de valor e estar satisfeito consigo mesmo (Dias \& Fontaine, 2001) ou como o modo como cada um se avalia ou se sente em relação a si mesmo (Simões \& Serra, 1987). Segundo Erdogan, Bauer, Truxillo e Mansfield (2012), a autoestima é o constructo mais utilizado no que concerne aos pensamentos que o sujeito tem de si mesmo, constructo este que integra sentimentos de valor ato-referenciados. Assim, a pessoa estabelece uma relação de identificação com seu trabalho e, ao perdê-lo, essa identificação será perdida e a autoestima, diminuída.

Tal como a autoestima, a satisfação com a vida é uma variável que condiciona a percepção dos sujeitos em relação ao mundo e a si mesmos (Haybron, 2008). A satisfação com a vida resulta de julgamentos globais construídos a partir de informações que se tornaram extremamente relevantes e conguentes para o sujeito (Galinha, 2008) e de informações recentes, associadas a fatores situacionais. Seu uso depende tanto de aspectos de personalidade como de condicionantes de ordem cultural (Kim-Prieto, Diener, Tamir, Scollon, \& Diener, 2005).

A satisfação com a vida se traduz no componente cognitivo do bem-estar subjetivo (Schimmack, 2008) e reflete a avaliação global que o sujeito faz da sua vida como um todo (Erdogan, Bauer, Truxillo, \& Mansfield, 2012; Pavot \& Diener, 1993), da forma como ela decorre, do contentamento subjacente no presente, da satisfação com o passado e ainda em relação ao desejo de mudar.

Sendo o desempenho da atividade profissional uma das tarefas associadas ao desenvolvimento na vida adulta, nosso propósito é explorar a relação entre a situação de desemprego e as avaliações que o sujeito faz de si mesmo (autoestima) e da sua vida como um todo (satisfação com a vida), principalmente quando medidas específicas de proteção social proporcionadas pelo RSI existem. Nesse sentido, apresentamos um estudo correlacional, de natureza quantitativa, com caráter exploratório, através do qual pretendemos relacionar a autoestima à satisfação com a vida; a idade dos beneficiários de RSI, o tempo de desemprego e da aplicação de medidas de proteção social (RSI) à autoestima e à satisfação com a vida; a idade ao tempo de desemprego e de benefício; e o tempo de desemprego ao tempo de RSI.

\section{MÉTODO}

\section{Participantes}

O método de amostragem selecionado foi não probabilístico e sua intencionalidade sustentou-se na acessibilidade aos sujeitos à pesquisa. A amostra foi constituída por 80 adultos caucasianos, beneficiários do RSI, sendo 62 do sexo feminino (77,5\%), e 18, do sexo masculino (22,5\%).

Com relação à idade dos participantes, registrou-se a média de 43,4 anos, com desvio-padrão de 9,3; a idade mínima foi de 22 anos, e a máxima, 63. No que diz respeito ao estado civil, 73,8\% dos sujeitos da amostra estavam inseridos na categoria casado ou união estável. Apenas $11,3 \%$ dos participantes é divorciado e tanto os solteiros como os viúvos apresentaram porcentagens equilibradas, na ordem dos 7,5\% dos beneficiários.

Relativamente à escolaridade, grande parte dos participantes da amostra $(53,8 \%)$ concluiu apenas o nível de ensino fundamental. Trinta por cento concluiu o $2^{\circ}$ ciclo do ensino básico e $11,3 \%$ tem o $3^{\circ}$ ciclo do ensino 
básico. O ensino médio só foi concluído por 2,5\% dos participantes e igual porcentagem diz respeito aos participantes sem escolaridade.

O conjunto familiar dos beneficiários do RSI acompanhados pelo Gabinete de Inserção Profissional (GIP) é, em média, constituído por 3,2 indivíduos, com desvio-padrão de 1,1, variando entre 1 e 6 sujeitos. Com relação ao desemprego, 93,8\% dos indivíduos indicaram que não era a primeira vez que se encontravam nessa situação.

Relativo ao número de meses em situação de desemprego, a variação foi entre 7 e 300 . Em média, o número de meses em situação de desemprego foi de 85.84 , com desvio-padrão de 66 . O número de meses de atribuição de RSI situou-se entre 2 e 204, com média de 48,8 meses e desvio-padrão de 49.

\section{Instrumentos}

Para avaliação da autoestima, optamos pela Rosenberg Self-Esteem Scale (Rosenberg, 1965) por se tratar de um instrumento de pequena dimensão (com apenas dez itens) e ser um dos mais utilizados para a avaliação da autoestima global. O construto é definido pelo autor como a avaliação que a pessoa faz e geralmente mantém sobre si mesma, a qual implica em um sentimento de valor, com um componente predominantemente afetivo, e expressão em atitudes de aprovação ou desaprovação em relação a si. Os sujeitos posicionam-se em uma escala Likert de quatro opções, do "concordo totalmente" ao "discordo totalmente".

Embora estudos como o de Romano, Negreiros e Martins (2007) equacionem uma estrutura bifatorial na validação da escala para contextos portugueses particulares, optamos pela versão portuguesa de Santos e Maia (1999), realizada a partir de uma amostra de 345 estudantes do Ensino Médio pertencentes ao $11^{\circ}$ e $12^{\circ}$ anos de escolaridade de quatro escolas públicas da cidade do Porto, com média de idade de 16,18 anos ( $D P=1,1)$ e equilibrada quanto ao sexo (a porcentagem de indivíduos do sexo masculino e feminino foi, respectivamente, $47 \%$ e $53 \%)$.

Essa opção se sustentou pelo fato de os autores corroborarem, em sua validação, com a estrutura unifatorial da escala original, assim como pelas boas qualidades psicométricas evidenciadas na referida validação, das quais se destaca a consistência interna (de 0,86$)$ e os índices de ajustamento resultantes da análise fatorial confirmatória da escala (RMSEA=0,08; CFI=0,90). Elas revelam seu caráter unitário, com a emergência de um fator único, explicativo da maior parte da variância. Os coeficientes de correlação que emergiram do padrão de correlações com outras variáveis relacionadas à autoestima foram ao encontro do esperado do ponto de vista teórico. Assim, a validade e a fidelidade do instrumento mostram-se adequadas, e, como tal, recomendável no contexto português.

Na Escala de Satisfação com a Vida (Diener, Emmons, Larsen \& Griffin, 1985), os cinco itens que a integram são de natureza global, permitindo aos sujeitos ponderar sobre os domínios de suas vidas em função de seus próprios valores e chegar a uma avaliação global de sua satisfação com a vida (Pavot \& Diener, 1993). Essa avaliação reflete uma perspectiva de longo prazo, assim como valores e objetivos conscientes.

A Escala de Satisfação com a Vida figura entre os instrumentos mais úteis e psicometricamente válidos. Foi validada pela primeira vez em Portugal por J. F. Barros e A. Barros (1990), tendo-se encontrado um alpha de Cronbach de 0,78 e vários índices de validade, como correlações significativas com outros construtos relevantes. A análise fatorial sublinhou o caráter unitário do construto, com a emergência de um fator único, explicativo da maior parte da variância.

A validação posterior da escala realizada por Simões (1992) reduziu o número de alternativas de resposta para cinco (de "discordo totalmente" a "concordo totalmente"), e apurou as questões da linguagem, em termos de tradução, tornando o conteúdo dos itens mais inteligível. Os resultados obtidos situam-se entre 5 e 25 , da menor para a maior satisfação com a vida, respectivamente. A escala apresenta bons índices de validade preditiva e, da análise fatorial, surge um fator único, que explica 53,1\% da variância total (com saturações de 0,77 para o item 1; 0,71 para o item 2; 0,79 para o item 3; 0,69 para o item 4; e 0,67 para o item 5). A fidelidade da escala avaliada pelo alpha de Cronbach revelou-se satisfatória, com valor de 0,77 . Tal estudo contou com 74 alunos da Faculdade de Psicologia e Ciências da Educação da Universidade de Coimbra e 130 adultos de diversas idades (média de idade de 40,89 anos), profissões e classes sociais.

Os resultados da validação de Simões (1992) são praticamente coincidentes com os da validação de Neto, Barros e Barros (1990), em que os índices de validade, na sua validação, são ainda mais elevados e mais próximos dos da escala original (Ribeiro, 2007), razão pela qual optamos por essa versão. 


\section{Procedimento de Coleta de Dados}

A coleta de dados foi realizada em um Gabinete de Inserção Profissional (GIP) da área metropolitana do Porto (Portugal). O GIP é um espaço que acompanha e auxilia desempregados na procura ativa de emprego, com o intuito de inseri-los ou reinseri-los no mundo laboral. Essa estrutura trabalha em conjunto com o centro de emprego local a fim de responder às necessidades da população, e está inscrita no Instituto de Emprego e Formação Profissional (IEFP). Após autorização da presidente da Junta de Freguesia ${ }^{1}$, na qual se enquadra o $\mathrm{GIP}^{2}$, para a coleta de dados, os sujeitos colaboraram, de forma facultativa, na resposta às perguntas do inquérito, tendo-lhes sido garantido o anonimato e a confidencialidade dos resultados. A aplicação dos instrumentos de avaliação foi realizada individualmente e em regime presencial.

\section{Procedimento de Análise de Dados}

Recolhidos os dados, procedeu-se à sua codificação, com a construção de uma base de dados, os quais foram submetidos a análises quantitativas com o recurso do SPSS (Social Package for Social Sciences) - Versão 19.0. Foram analisadas as medidas de tendência central (média e desvio-padrão) para as variáveis autoestima e satisfação com a vida. Para as análises correlacionais, foi utilizado o coeficiente de correlação de Pearson.

\section{RESULTADOS}

Em termos de estatística descritiva, a média da autoestima da amostra foi de 2,95. No estudo de validação da versão portuguesa da escala de autoestima de Rosenberg (Santos \& Maia, 2003) a média foi de 3,14, com desvio-padrão de 4,86.

A média da satisfação com a vida foi de 1,85, valor baixo considerando os resultados médios de outros estudos de validação da versão portuguesa da escala de satisfação com a vida (Neto, Barros \& Barros, 1990; Simões, 1992).

Apresentamos a seguir a Tabela 1, relativa às correlações das variáveis em estudo.

TABELA 1. Matriz de correlações

\begin{tabular}{|c|c|c|c|c|c|}
\hline & Autoestima & $\begin{array}{l}\text { Satisfação com } \\
\text { a vida }\end{array}$ & Idade & $\begin{array}{l}\text { Tempo de } \\
\text { desemprego } \\
\text { (em meses) }\end{array}$ & $\begin{array}{l}\text { Tempo de prote- } \\
\text { ção social - RSI } \\
\text { (em meses) }\end{array}$ \\
\hline Autoestima & & $\begin{array}{l}0,40^{\star *} \\
0,00\end{array}$ & $\begin{array}{r}-0,19 \\
0,07\end{array}$ & $\begin{array}{c}-0,23^{\star} \\
0,03\end{array}$ & $\begin{array}{r}-0,13 \\
0,22\end{array}$ \\
\hline Satisfação com a vida & & & $\begin{array}{r}-0,05 \\
0,66\end{array}$ & $\begin{array}{r}-0,12 \\
0,26\end{array}$ & $\begin{array}{r}-0,12 \\
0,28\end{array}$ \\
\hline Idade & & & & $\begin{array}{l}0,53^{\star *} \\
0,00\end{array}$ & $\begin{array}{l}0,48^{* *} \\
0,00\end{array}$ \\
\hline Tempo de desemprego (em meses) & & & & & $\begin{array}{l}0,87^{\star *} \\
0,00\end{array}$ \\
\hline $\begin{array}{l}\text { Tempo de proteção social - RSI } \\
\text { (em meses) }\end{array}$ & & & & & \\
\hline
\end{tabular}

Nota. * Correlação significativa para um nível de confiança de 95\%; ** Correlação altamente significativa para um nível de confiança de $99 \%$.

Quando relacionamos a autoestima com a satisfação com a vida, verificamos que à medida que a primeira aumenta, o mesmo acontece com a segunda. Essa correlação $(r=0,40)$ é estatisticamente muito significativa $(p=0,00)$. Os resultados inscritos na Tabela 1 nos permitem concluir que não existem diferenças estatisticamente significativas quando relacionamos a idade dos sujeitos com a autoestima ou com a satisfação com a vida.

Quando relacionamos o número de meses em situação de desemprego com a autoestima, é possível perceber que há correlação negativa $(r=-0,23)$ e significativa $(p=0,03)$. Ou seja, à medida que a situação de desemprego perdura, mais baixa é a autoestima dos indivíduos. Por outro lado, quando correlacionamos o número de meses

1 No Brasil, a Junta de Freguesia tem como equivalente a Prefeitura.

2 Aproveitamos para agradecer à Junta de Freguesia de Canelas - Vila Nova de Gaia - Portugal, pela autorização e colaboração na coleta da amostra. 
em situação de desemprego com a satisfação com a vida, não encontramos valores estatisticamente significativos, assim como não são registrados valores significativos quando correlacionamos o número de meses de atribuição do RSI com a autoestima e com a satisfação com a vida.

Registra-se, porém, correlação positiva $(r=0,53)$ e altamente significativa $(p=0,00)$ entre a idade dos sujeitos e o tempo de desemprego, bem como correlação positiva $(r=0,48)$ e altamente significativa $(p=0,00)$ entre a idade e o tempo de atribuição de RSI. Por último, não é surpreendente a correlação positiva $(r=0,07)$ e altamente significativa $(p=0,00)$ encontrada entre o tempo de desemprego dos sujeitos e o tempo atribuição de RSI.

\section{DISCUSSÃO}

Os resultados obtidos mostram que a autoestima dos indivíduos que se encontram em situação de desemprego situa-se abaixo das médias registradas em estudos semelhantes, mas, ainda assim, dentro de um desvio-padrão aceitável. O mesmo não acontece com a satisfação com a vida, cuja média é mais baixa que em outros estudos e revela insatisfação com a vida em geral. Sendo o trabalho uma dimensão central na vida dos sujeitos adultos, seria esperado que a situação de desemprego alterasse negativamente a satisfação com a vida. Essa alteração não é tão evidente quando está em questão a autoestima dos desempregados que, apesar de diminuída, parece, ainda assim, preservada. A partir de tais observações decorrentes da análise da avaliação dos conceitos em estudo por meio da presente amostra, e considerando suas especificidades, analisaremos os resultados desta investigação, a fim de ir ao encontro de cada um dos objetivos específicos formulados.

A correlação que observamos entre autoestima e satisfação com a vida na amostra de desempregados em situação de proteção social vai ao encontro da literatura, sobretudo de outros estudos focados em outras populações e contextos. Por exemplo, o estudo de Diener (1984) aponta para uma elevada correlação entre autoestima e satisfação com a vida, mas, no que diz respeito à satisfação com o domínio do trabalho, a correlação é moderada. Em outros domínios da satisfação com a vida, como a satisfação com a saúde e com a comunidade, a correlação com a autoestima é ainda mais baixa. Parece, então, que a articulação verificada declara-se de forma mais evidente quando se reporta à satisfação da vida como um todo, tal como foi avaliada em nosso estudo. Assim, existe uma conexão entre a satisfação com a vida e a autoestima, na medida em que nos sentimos responsáveis pela forma como decorre a nossa vida, ou na medida em que nossa identidade é constituída, em parte, por nossas histórias de vida (Haybron, 2007).

Nossos resultados corroboram também às conclusões de Santos e Maia (2003), pois tudo indica que os sujeitos que apresentam maior nível de autoestima exprimem maior satisfação com a vida. Essa relação entre conceitos pode ter sua origem, tal como sugerem Cummins e Nistico (2002), na motivação que os indivíduos têm para preencher a necessidade de autoestima, a fim de obter sucesso e evitar o fracasso em diferentes domínios, levando-os a regular suas metas e ações de forma contingente com o valor que atribuem a si mesmos (Crocker, Brook, Niiya, \& Villacorta, 2006).

Dessa forma, Cummins e Nistico (2002) pressupõem que a satisfação com a vida partilha dos mesmos mecanismos da autoestima em três tipos de crenças que se relacionam com a autopromoção, com o controle percebido e com o otimismo, pilares fundamentais do bem-estar subjetivo. Efetivamente, alguns estudos, como o de Gómez, Posada, Barrera e Cruz (2007), verificaram que a satisfação com alguns domínios específicos da vida, sobretudo a saúde, os relacionamentos afetivos e o trabalho, assim como as variáveis do sistema do self, em particular a autoeficácia, têm papel preditor no bem-estar subjetivo. Haybron (2007) admite que elevados níveis de satisfação com a vida encontrados em outros estudos refletem, em parte, ideais de gratidão e até mesmo autoestima, e não tanto o bem-estar em termos de condições de vida.

Para Heatherton e Polivy (1991), o componente estável da autoestima é determinado em grande parte por sentimentos de competência e de realização. Erdogan et al. (2012) reportam-se a Crocker e Park (2004) a este propósito. Estes autores sugerem que os indivíduos que procuram incrementar o seu nível de autoestima fazem-no não apenas para experienciar um sentimento de competência, mas também para alcançar um sentido de controlo. Por outras palavras, sentimentos de competência e de controlo sobre a vida própria são aspetos importantes dos sentimentos de autovalorização.

Em nosso estudo, registramos uma correlação negativa entre a autoestima e o tempo de desemprego dos beneficiários de RSI, mas não encontramos qualquer correlação significativa no que concerne à satisfação com a vida. A correlação negativa entre a autoestima e o número de meses em que o sujeito se encontra desem-

Rev. Psicol., Organ. Trab., abr-jun 2015, vol. 15 num. 2 
pregado corrobora os resultados de Cruz (2009). É esperado que, à medida que o tempo passe, a situação de desemprego influencie negativamente na avaliação que o sujeito faz de si mesmo, não apenas pela situação de desemprego e pelas implicações financeiras e sociais que comporta, mas, sobretudo, pelas representações sociais negativas e estereotipadas associadas à situação de desemprego. Sentimentos de inutilidade, quebra de rotina, perda de estatuto social, entre outros, podem contribuir como possíveis elementos significativos dessa relação.

No que diz respeito à satisfação com a vida, a questão temporal parece ser menos determinante. Estudos como os de Steger e Kashdan (2007) mostram que a satisfação com a vida é moderadamente estável ao longo do tempo, pelo menos por cerca de um ano. Parker, Martin e Marsh (2008) relacionam a satisfação com a vida a aspectos de personalidade, sublinhando justamente o seu caráter estável. Efetivamente, em seu estudo, algumas dimensões do autoconceito aparecem como elementos mediadores na relação entre os aspectos de personalidade e a satisfação com a vida. Quando longos períodos de tempo estão em questão (por exemplo, 17 anos, como no estudo de Fujita \& Diener, 2005), a estabilidade da satisfação com a vida é modesta, e parte dos sujeitos (cerca de $25 \%$ da amostra constituída no referido estudo) apresentam mudanças significativas com relação à satisfação com a vida.

Outro argumento que podemos avançar para sustentar a (in)existência de correlação entre cada um desses conceitos em análise prende-se com a sua natureza. De fato, a autoestima é componente claramente afetivo do self, que se traduz em julgamentos de valor acerca de si mesmo, enquanto a satisfação com a vida é de natureza cognitiva. Admite-se que a situação de desemprego estabeleça uma relação mais evidente e significativa com dimensões afetivas do self e menos com dimensões imbuídas de processos de racionalização. De resto, nosso resultado não é inédito na literatura. Argolo e Araújo (2004), em um estudo centrado no impacto do desemprego sobre o bem-estar psicológico dos trabalhadores, não encontraram diferenças significativas da deterioração dedesse construto (ao qual se pode também reportar a satisfação com a vida) entre os desempregados da amostra, aferida pelo tempo de desemprego.

No que diz respeito à idade dos beneficiários de RSI e os níveis de autoestima, nossos resultados são congruentes com os de Cruz (2009) ao não ser encontrado qualquer tipo de correlação significativa. Também não se verifica qualquer correlação entre a idade dos sujeitos e a satisfação com a vida. Considerando a média de idades da amostra, temos em sua maioria sujeitos que se encontram na adultícia madura. As mudanças que se operam no sistema do self são muito graduais e sutis. Alguns estudos referidos reportam-se ao autoconceito, mas sendo a autoestima o componente avaliativo deste construto, entendemos ser desejável citá-los. Por exemplo, o estudo de Marsh, Smith, Barnes e Butler (1983) avaliou a estabilidade versus a mudança em sete áreas diferentes do autoconceito de pré-adolescentes durante de aproximadamente seis meses. Nele, podemos encontrar argumentos que sustentam tanto a estabilidade como a mudança do autoconceito. A mudança representa o outro lado da estabilidade. É evidente que o auto-conceito não é completamente estável e é permeável aos acontecimentos de vida dramáticos ou a intervenções sistemáticas. Para Marsh et al. (1983), é justamente aqui que reside o dilema.

Segundo Neuenschwander (2002), os elementos centrais do autoconceito são estáveis, e aqueles mais periféricos são mais suscetíveis à mudança. "Sendo o autoconceito relativamente estável, os acontecimentos de vida podem ter um impacto específico em dimensões particulares do autoconceito" (Marsh et al., 1983, p. 788). Para Handel (1987), existe uma consistência fenomenológica subjacente aos julgamentos autopercebidos de estabilidade e mudança, verificada por meio da inter-relação entre as autoavaliações atuais do sujeito e as que resultam das autoavaliações retrospectivas. As mudanças, quando registradas pelo indivíduo, são vistas como positivas, e, raramente, como negativas, mas nunca percebidas como drásticas.

Por outro lado, existe correlação entre a quantidade de mudança percebida pelo sujeito e a importância relativa de cada uma delas. Observa-se ainda uma percepção subjetiva de maior autoconhecimento, maior autoconfiança e maior capacidade de autocontrole. Os indivíduos que se percebem como mais estáveis no tempo indicam menos acontecimentos e experiências de vida críticos e apresentam nível mais baixo de complexidade cognitiva na construção da representação de si mesmos e dos outros significativos. Apesar de manifestarem nível mais elevado de bem-estar e satisfação, têm maior necessidade de aprovação por parte dos outros, o que nos leva a pensar que estão mais centrados nas avaliações externas que os outros possam fazer de si do que propriamente de processos reflexivos dirigidos ao self. 
A tendência para procurar aprovação dos outros por meio da adesão a normas desejáveis e socialmente partilhadas pode ser vista como mais uma tentativa de atribuir continuidade ao self. Quanto mais negativa for a avaliação das mudanças retrospectivas, mais baixo o nível de autoestima, tanto no atual momento como em sua futura projeção. As autoavaliações em relação às mudanças operadas no self dependem das autopercepções e de sua importância relativa, assim como da relevância da autopercepção de mudança. Assim, os sujeitos com elevada autoestima apresentam elevados níveis nas faces de autoconceito percebidas como mais importantes, mas os níveis de importância atribuídos não contribuem para a predição de sua autoestima (Marsh, 1986).

Apesar da autoestima se reportar especificamente ao componente avaliativo do autoconceito, um estudo longitudinal de dez anos permitiu a comparação de padrões que destacam clara evidência para uma invariância estrutural de dimensões do autoconceito (Marsh, 1994). No estudo de Block e Robins (1993), observa-se também um tipo de estabilidade centrado na ordenação dos atributos de cada sujeito ao longo do tempo. Os níveis de autoconceito mais elevados no início também foram os mais elevados adiante. Esse dado destaca a existência de uma consistência intra-individual na forma como cada sujeito se percebe ao longo do tempo.

Não parece ser evidente uma interação entre a dimensão temporal e as variáveis situacionais, pois a possível evolução positiva que se registra quanto à percepção e à avaliação que os sujeitos fazem de si mesmos resultará de um processo desenvolvimental, pouco influenciável pelas mudanças contextuais.

Visto todos esses processos equilibratórios e compensadores, compreende-se que a idade não estabelece relação significativa com a autoestima. No que diz respeito à satisfação com a vida, a estabilidade, já referida, parece também servir de argumento para a ausência de qualquer correlação com a idade dos sujeitos nesse contexto (Simões et al., 2000; Simões et al., 2003).

Quando investigada a possível relação entre a aplicação de medidas de proteção social (RSI) e a autoestima e a satisfação com a vida, verifica-se ausência de correlações entre as medidas de proteção social (RSI) e os dois construtos em estudo. Tais resultados podem ser compreendidos pelo fato de o apoio em questão se traduzir em recursos para suprir necessidades básicas por meio de uma ajuda financeira e favorecimento da progressiva inserção social e profissional.

Talvez a eficácia dessas medidas não seja suficiente para os objetivos a que se propõem, principalmente na promoção da satisfação com a vida. Esta amostra reporta-se a um conjunto de pessoas em vulnerabilidade social, em que o baixo nível de escolaridade parece constituir um elemento que dificulta o processo de reinserção social.

A correlação positiva encontrada entre a idade e o tempo de desemprego e a idade e o tempo de proteção social nos leva a concluir que a idade é, de fato, um aspecto que dificulta a reinserção no mercado de trabalho e a perpetuação da situação de desemprego gera exclusão social. O desemprego de exclusão, definido por Clavel (2004), se traduz no desemprego de longa duração, considerado quando o desemprego se prolonga por mais de um ano. Essa situação atinge indivíduos com mais de 25 anos (Clavel, 2004), os quais mais se beneficiam do serviço de proteção social. Tal fato justifica, em nosso estudo, a correlação positiva e significativa encontrada entre o tempo de desemprego e o tempo em que são aplicadas as medidas de proteção social.

$\mathrm{Na}$ confrontação dos resultados com os contributos de outros autores, entendemos, à semelhança de Vatin (2002), ser necessário reestruturar alguns sistemas por meio de medidas que podem passar pela redução do número de horas de trabalho, pela reorganização das estruturas e atividades sociais e pela melhoria do ambiente laboral dos sujeitos, com a otimização de seus recursos.

Sabemos que a vivência de bem-estar é especialmente devastadora nos desempregados, não se verificando fenômenos idênticos nos aposentados ou nas donas de casa (Galinha, 2008). Sabemos também que menores níveis de bem-estar pessoal subjetivo estão associados a maior desigualdade no sistema social. “...o bem-estar não tem só uma dimensão individual, mas também societal (comporta avaliações sobre o sistema e não apenas sobre os indivíduos), e essa dimensão societal cobre, à semelhança da dimensão individual, aspectos objetivos e subjetivos" (Vala, 2013, p. 30). Falamos no bem-estar porque a satisfação com a vida é uma de suas dimensões, e a autoestima é condição para sua experiência.

As medidas de proteção social, discutíveis em termos da eficácia como medida de combate ao desemprego têm a mais-valia de poder criar sociabilidades, que de outra forma esses sujeitos não teriam, e de contribuir para a diminuição do risco de exclusão social em que tais indivíduos já se encontram. A criação de laços, a comparação social e os processos de partilha são exemplos de mecanismos que se inscrevem nessa filosofia de redução de risco. Todavia, a operacionalização dessas medidas não parece ter repercussões efetivas, principal- 
mente nas que referem-se à autoestima dos desempregados de longa duração que, por essa condição, veem os apoios se perpetuarem em termos de proteção social que não parecem ter capacidade para reverter a situação de desemprego.

Esta investigação, por sua natureza correlacional, não permite estabelecer relações de causa e efeito entre as variáveis em análise. Nesse sentido, sugere-se estudos com procedimentos metodológicos mais sofisticados, especialmente com o recurso de análise de regressão e com caráter longitudinal, para conhecimento mais aprofundado do impacto da situação de desemprego ao longo do tempo.

No entanto, este estudo pode constituir-se como mais um contributo para a investigação dess fenômeno na região Norte de Portugal e principalmente em Vila Nova de Gaia, servindo de referencial para análise por parte das instituições e organizações locais, cuja função passa pela atenuação dos efeitos psicossociais do desemprego. Esse é um contributo premente, a avaliar pela taxa de desempregados inscritos nos centros de emprego e formação profissional no total da população residente entre 15 e 64 anos. Segundo a base de dados Portugal Contemporâneo, Pordata (n.d.b), essa taxa é de 10,3\% em Portugal Continental, 11,9\% no Norte do país e 16\% no município de Vila Nova de Gaia, pelo que este estudo se apresenta como uma mais valia na análise do fenômeno em um dos municípios portugueses mais afetados pelo flagelo do desemprego.

\section{REFERÊNCIAS}

Alcántara, J. (1997). Como educar a autoestima? Lisboa: Plátano Edições Técnicas

Álvaro J. (1992). Desempleo y bienestar psicológico. Madrid: Siglo XXI.

Álvaro, J., \& Garrido, A. (2002). Reflexos da situação laboral na saúde mental dos jovens. In A. Mendes, L. Borges, \& M., Ferreira (Orgs.), Trabalho em transição, saúde em risco. Brasília: UnB.

Álvaro, J., Torregrosa, J., \& Luque, A. (1992). Estructura social e saúde mental. In J. Álvaro, J. Torregrosa, \& A. Luque (Orgs.), Influencias sociais e psicológicas en la salud mental (pp. 9-30). Madrid: Siglo XXI.

Argolo, J., \& Araújo, M. (2004). O impacto do desemprego sobre o bem-estar psicológico dos trabalhadores da cidade de Natal. Revista de Administração Contemporânea, 8(4), 161-182.

Banks, M. (1992). Desempleo y salud mental: Investigaciones británicas recientes. In J. Álvaro, J. Torregrosa, \& A. Luque (Orgs.), Influencias sociais e psicológicas en la salud mental (pp. 143-170). Madrid, Siglo XXI.

Blanch, J. (1990). Del viejo al nuevo paro. Un análisis psicológico e social. Barcelona: Promociones y Publicaciones Universitarias, S.A.

Block, J., \& Robins, R. (1993). A longitudinal study of consistency and change in self-esteem from early adolescence to early adulthood. Child Development, 64, 909-923.

Buendía, J. (2001). Desempleo y salud mental. In J. Buendía \& F. Ramos (Orgs.), Empleo estrés y salud. Madrid: Edições Pirâmide.

Caleiro, A. (2012). Unemployment and happiness in Portugal. In A. Bento-Gonçalves \& A. Vieira (Eds.), Portugal: Economic, political and social issues (pp. 113-123). New York: Nova Science Publishers.

Carvalho, M. (2008). Desemprego. São Paulo: Faculdade Piauiense de Processamento de Dados.

Cavalcanti, B. (2007). Autoestima, importante fator emocional no mundo das organizações. Recife: Universidade Católica de Pernambuco.

Clavel, G. (2004). A sociedade da exclusão - Compreendê-la para dela sair. Porto: Porto Editora.

Costa, A. (1998). Exclusões sociais. Lisboa: Gradiva.

Costa, A.; Baptista, I.; Perista, P., \& Carrilho, P. (2008). Um olhar sobre a pobreza - Vulnerabilidade e exclusão social no Portugal contemporâneo. Lisboa: Gradiva.

Crocker, J., Brook, A., Niiya, Y., \& Villacorta, M. (2006). The pursuit of self-esteem: Contingencies of self-worth and selfregulation. Journal of Personality, 74, 1749-1772. doi: 10.1111/j.1467-6494.2006.00427.x

Cruz, M. (2009). O impacto do desemprego nos níveis da depressão, autoestima e desânimo aprendido - estudo comparativo numa amostra de empregados e desempregados (Tese de mestrado não publicada), Universidade Fernando Pessoa. Porto.

Cummins, R. A., \& Nistico, H. (2002). Maintaining life satisfaction: The role of positive cognitive bias. Journal of Happiness Studies, 3, 37-69. 
Dias, M., \& Fontaine, A. (2001). Tarefas desenvolvimentais e bem-estar de jovens universitários. Lisboa: Fundação Calouste Gulbenkian.

Diener, E. (1984). Subjective well-being. Psychological Bulletin, 95(3), 542-575.

Diener, E., Emmons, R.. Larsen, R., \& Griffin, S. (1985). The satisfaction with life scale. Journal of Personality Assessment, 49(1), 71-75.

Erdogan, B., Bauer, T., Truxillo, D., \& Mansfield, L. (2012). Whistle while you work: A review of life satisfaction literature. Journal of Management, 38(4), 1038-1083. doi: 10.1177/0149206311429379

Fujita, F., \& Diener, E. (2005). Life satisfaction set point: Stability and change. Journal of Personality and Social Psychology, 88(1), 158-164. doi: 10.1037/0022-3514.88.1.158

Galinha, I. (2008). Bem-Estar subjectivo: Factores cognitivos, afectivos e contextuais. Coimbra: Quarteto.

Gómez, V., Posada, C., Barrera, F., \& Cruz, J. (2007). Factores predictores de bienestar en una muestra colombiana. Revista Latinoamericana de Psicología, 39(2), 311-325.

Handel, A. (1987). Perceived change of self among adults: A conspectus. In T. Honess \& K. Yardley (Eds.), Self and identity: Perspectives across lifespan. London: Routledge \& Kegan Paul.

Haybron, D. (2007). Life satisfaction, ethical reflection, and the science of happiness. Journal of Happiness Studies, 8, 99-138. doi: 10.1007/s10902-006-9006-5

Haybron, D. (2008). Philosophy and science of subjective well-being In M. Eid \& R. J. Larsen (Eds.), The science of subjective well-being (pp. 17-43). New York: The Guilford Press.

Heatherton, T., \& Polivy, J. (1991). Development and validation of a scale for measuring state self-esteem. Journal of Personality and Social Psychology, 60, 895-910. doi: 10.1037/0022-3514.60.6.895

Janeiro, I., \& Marques, J. (1999). Autoestima e atitudes de planejamento e de exploração da carreira em jovens do $9^{\circ}$ e $11^{\circ}$ anos. Revista Portuguesa de Psicologia, 34, 83-99.

Kim-Prieto, C., Diener, E., Tamir, M., Scollon, C., \& Diener, M. (2005). Integrating the diverse definitions of happiness: A timesequential framework of subjective well-being. Journal of Happiness Studies, 6, 261-300. doi: 10.1007/s10902-005-7226-8

Lei $n^{\circ} 7$ de 12 de fevereiro de 2009 (2009). Aprova a revisão do Código do Trabalho. Recuperado de http://www.cite.gov.pt/pt/ legis/Lei007_2009.html

Lei $n^{\circ} 53$ de 14 de outubro de 2011 (2011). Procede à segunda alteração ao Código do Trabalho, aprovado em anexo à Lei . $^{\circ}$ 7/2009, de 12 de Fevereiro, estabelecendo um novo sistema de compensação em diversas modalidades de cessação do contrato de trabalho, aplicável apenas aos novos contratos de trabalho. Recuperado de http://www.cite.gov.pt/asstscite/ downloads/legislacao/Lei_53_2011.pdf

Lei $n^{\circ} 23$ de 25 de junho de 2012 (2012). Procede à terceira alteração ao Código do Trabalho, aprovado pela Lei n. ${ }^{\circ}$ 7/2009, de 12 de fevereiro. Recuperado de http://www.dgaep.gov.pt/upload/Legis/2012_I_23_25_063.pdf

Lei $n^{\circ}$ 13/2003 de 21 de maio de 2003 (2003). Revoga o rendimento mínimo garantido previsto na Lei n.o 19-A/96, de 29 de Junho, e cria o rendimento social de inserção. Recuperado de http://www.riac.azores.gov.pt/NR/rdonlyres/BC22539A-60594053-B9DB-241455F92A33/133141/Lei_13_2003.pdf

Luque, A. (1992). Consecuencias psicosociales de las transiciones de los jóvenes a la vida activa (Tese de doutorado não publicada). Faculdade de Psicologia da Universidade Complutense de Madrid, Madrid.

Machado, H. (2003). A identidade e o contexto organizacional: Perspectivas de análise. Revista de Administração Contemporânea, 7, 51-73.

Marsh, H. (1986). Global Self-esteem: Its relation to specific facets of self-concept and their importance. Journal of Personality and Social Psychology, 51(6), 1224-1236. doi: 10.1037/0022-3514.51.6.1224

Marsh, H. (1994). Using the national longitudinal study of 1988 to evaluate theoretical models of self-concept: The Self-description Questionnaire. Journal of Educational Psychology, 86(3), 439-456.

Marsh, H., Smith, I., Barnes, J., \& Butler, S. (1983). Self-concept: Reliability, stability, dimensionality, validity, and the measurement of change. Journal of Educational Psychology, 75(5), 772-790.

Neto, F.; Barros, J. F. \& Barros, A. (1990). Satisfação com a vida. In L. Almeida, R. Santiago, P. Silva, O. Caetano, \& J. Marques (Eds.), A acção educativa: Análise psico-social. Leiria: ESEL/APPORT.

Neuenschwander, M. (2002). Desenvolvimento e identidade na Adolescência. Coimbra: Almedina. 
Parker, P., Martin, A., \& Marsh, H. (2008). Factors predicting life satisfaction: A process model of personality, multidimensional self-concept, and life satisfaction. Australian Journal of Guidance \& Counselling,18(1), 15-29. doi: 10.1375/ajgc.18.1.15

Pavot, W., \& Diener, E. (1993). Review of the satisfaction with life scale. Psychological Assessment, 5(2), 164-172.

Pereira, M., \& Brito, M. (2006). Desemprego e subjetividade no contexto brasileiro: Uma análise interpretativa sob a ótica dos excluídos do mercado de trabalho industrial. Revista Mal-estar e Subjetividade, 6(1), 143-181.

Pordata (n.d.a). Números de Portugal. Recuperado de http://www.pordata.pt/Portugal/Summary+Table/Portugal-5452\#

Pordata (n.d.b). Desempregados inscritos nos centros de emprego e de formação profissional no total da população residente com 15 a 64 anos (\%). Recuperado de http://www.pordata.pt/Municipios/Desempregados+inscritos+nos+centros+de+empre go+e+de+formacao+profissional+no+total+da+populacao+residente+com+15+a+64+anos+\%28percentagem\%29-592

Ribeiro, J. (2007). Avaliação em psicologia da saúde: Instrumentos publicados em Português. Coimbra: Quarteto.

Ripoll, P., Rodriguez, I., \& Peiró, J. (1996). Desempleo. In J. Peiró \& F. Prieto (Orgs.), Tratado de psicología del trabajo: Aspectos psicosociales del trabajo (Vol. II, pp. 225-247). Madrid: Editorial Síntesis.

Romano, A., Negreiros, J., \& Martins, T. (2007). Contributos para a validação da escala de autoestima de Rosernberg numa amostra de adolescentes da região interior norte do país. Psicologia, Saúde \& Doenças, 8(1), 109-116.

Rosenberg, M. (1965). Society and adolescent self-image. Princeton: Princeton University Press.

Santos, P., \& Maia, J. (2003). Análise factorial confirmatória e validade preliminar de uma versão portuguesa da escala de autoestima de Rosenberg. Porto: Faculdade de Letras da Universidade do Porto.

Santos, P., \& Maia, J. (1999). Adaptação e análise factorial confirmatória da Rosenberg Self-esteem Scale com uma amostra de adolescentes: Resultados preliminares. In A. P. Soares, S. Araújo \& S. Caires (Orgs.), Avaliação Psicológica: Formas e Contextos (Vol. VI, pp. 101-103). Braga: APPORT.

Schimmack, U. (2008). The structure of subjective well-being. In M. Eid \& R. J. Larsen (Eds.), The Science of Subjective Wellbeing (pp.97-123). New York: The Guilford Press.

Sennett, R. (2000). La corrosión del carácter, las consecuencias personales del trabajo en el nuevo capitalismo. Barcelona: Anagrama.

Simões, A. (1992). Ulterior validação de uma escala de satisfação com a vida (SWLS). Revista Portuguesa de Pedagogia, 26(3), 503-515.

Simões, M., \& Serra, A. (1987). A importância do auto-conceito na aprendizagem escolar. Separata da Revista Portuguesa de Pedagogia, 21, 233-252.

Simões, A., Ferreira, J., Lima, M., Pinheiro, M., Vieira, C., Matos, A., \& Oliveira, A. (2000). O bem-estar subjectivo: Estado actual dos conhecimentos. Psicologia, Educação e Cultura, 4(2), 243-279.

Simões, A., Ferreira, J., Lima, M., Pinheiro, M., Vieira, C., Matos, A., \& Oliveira, A. (2003). O bem-estar subjectivo dos adultos: Um estudo transversal. Revista Portuguesa de Pedagogia, 37(1), 5-30.

Steger, M., \& Kashdan, T. (2007). Stability and specificity of meaning in life and life satisfaction over one year. Journal of Happiness Studies, 8, 161-179. doi: 10.1007/s10902-006-9011

Stiglitz, J. (2004). Globalização: A grande desilusão. Lisboa: Terramar.

Vala, J. (2013). Bem-estar. In J.L. Cardoso, P. M. \& J. M. Pais (Orgs.), Portugal de A a Z - Temas em aberto. Paço de Arcos: Impresa Publishing/Expresso.

Vatin, E. (2002). Epistemologia e sociologia do trabalho. Lisboa: Instituto Piaget.

Vieira, F. (2014). Impacto do desemprego no bem-estar psicológico (Dissertação de mestrado não publicada). Faculdade de Psicologia da Universidade Lusófona do Porto, Porto. 